\title{
A Space-Time Mixed Galerkin Marching-on-in-Time Scheme for the Time Domain Combined Field Integral Equation
}

\author{
Yves Beghein, Kristof Cools, Hakan Bağc1, and Daniël De Zutter, Fellow, IEEE
}

\begin{abstract}
The time domain combined field integral equation (TD-CFIE), which is constructed from a weighted sum of the time domain electric and magnetic field integral equations (TDEFIE and TD-MFIE) for analyzing transient scattering from closed perfect electrically conducting bodies, is free from spurious resonances. The standard marching-on-in-time technique for discretizing the TD-CFIE uses Galerkin and collocation schemes in space and time, respectively. Unfortunately, the standard scheme is theoretically not well understood: stability and convergence have been proven for only one class of spacetime Galerkin discretizations. Moreover, existing discretization schemes are non-conforming, i.e., the TD-MFIE contribution is tested with divergence conforming functions instead of curl conforming functions. We therefore introduce a novel spacetime mixed Galerkin discretization for the TD-CFIE. A family of temporal basis and testing functions with arbitrary order is introduced. It is explained how the corresponding interactions can be computed efficiently by existing collocation-in-time codes. The spatial mixed discretization is made fully conforming and consistent by leveraging both Rao-Wilton-Glisson and BuffaChristiansen basis functions and by applying the appropriate bi-orthogonalization procedures. The combination of both techniques is essential when high accuracy over a broad frequency band is required.
\end{abstract}

Index Terms-Marching-on-in-time, combined field integral equation, space-time Galerkin method, mixed discretization, Buffa-Christiansen functions.

\section{INTRODUCTION}

$\mathbf{T}$ $\mathrm{HE}$ time domain electric and magnetic field integral equations (TD-EFIE and TD-MFIE) belong to the family of retarded potential boundary integral equations (RP-BIEs), which are specifically constructed for analyzing transient scattering from perfect electrically conducting (PEC) bodies. Both the TD-EFIE and TD-MFIE, however, are susceptible to resonant instabilities, originating in internal resonances that are regime solutions to these equations. However, the time domain combined field integral equation (TD-CFIE), which is a weighted sum of the TD-EFIE and the TD-MFIE, does not support regime solutions, and thus is not susceptible to resonant instabilities [1].

The TD-CFIE usually is discretized using the standard marching-on-in-time (MoT) technique, which makes use of

Y. Beghein (email: yves.beghein@ugent.be) and D. De Zutter are with the Department of Information Technology (INTEC), Ghent University, Belgium

$\mathrm{K}$. Cools is with the University of Nottingham, Nottingham NG7 2RD, UK

$\mathrm{H}$. Bağc1 is with the King Abdullah University of Science and Technology (KAUST), 4700 KAUST, Thuwal 23955-6900, Kingdom of Saudi Arabia

The work of Y. Beghein was supported by a doctoral grant from the Agency for Innovation by Science and Technology in Flanders (IWT).
Galerkin and collocation schemes in space and time, respectively. First, the current density is approximated by an expansion in spatial and temporal basis functions (usually the Rao-Wilton-Glisson (RWG) functions [2] and the Lagrange interpolators [3], respectively), which is inserted in the TDCFIE. Next, the resulting equation is tested by a set of spatial testing functions (Galerkin-in-space) and evaluated at equidistant time steps (collocation-in-time). The temporal basis functions fulfil the discrete causality constraint, i.e. upon discretization, the TD-CFIE yields a system that can be solved for the unknown current expansion coefficients at successive time steps.

The solutions to this classically discretized TD-CFIE are free from resonant instabilities, but are often found to be less accurate compared to solutions to the frequency domain EFIE, which is regarded to be the benchmark when it comes to accuracy (away from resonant frequencies). The reasons for this are:

1) Although the classical discretization of the TD-CFIE is a spatial Galerkin scheme, it is a collocation-in-time scheme. This implies that it cannot be expected to benefit from the enhanced accuracy and convergence properties enjoyed by e.g. finite element methods.

2) The classical discretization of the TD-CFIE is spatially non-conforming in that the TD-MFIE contribution is tested by divergence conforming functions instead of curl conforming functions, and therefore yields inaccurate results. This has also been observed for both the frequency domain MFIE and CFIE [4], [5], [6].

In this contribution, a space-time mixed Galerkin scheme for discretizing the TD-CFIE is introduced. The resulting MoT scheme does not suffer from the problems listed above; it improves on the accuracy of the classical MoT scheme for the TD-CFIE by:

1) Introducing a higher order temporal mixed Galerkin scheme. For any order $p$, families of basis and testing functions for the temporal discretization of the TD-CFIE are presented. These families fulfil the discrete causality constraint and thus give rise to a discrete system amenable to marching-on-intime. Numerical results demonstrate that the accuracy of the resulting scheme is superior to that of the classical collocationin-time schemes.

2) Applying a conforming spatial Galerkin scheme. A conforming mixed discretization of the frequency domain MFIE, leveraging RWG and Buffa-Christiansen (BC) [7], [8] functions, has been introduced in [4]. It has also been applied 
to the frequency domain CFIE [5] and the frequency domain Calderón preconditioned CFIE [9]. This scheme is now applied to the TD-MFIE contribution in the TD-CFIE. This renders the discretization of both the TD-EFIE and the TD-MFIE contributions in the TD-CFIE conforming and well-tested. In the earliest implementations of spatially mixed frequency domain CFIEs [5], the RWG-RWG discretized EFIE and BCRWG discretized MFIE were simply added. This scheme is not consistent, because it adds RWG and BC tested values in the same equation. In [9], this inconsistency has been removed by applying a Calderón preconditioner to the EFIE contribution. Here, consistency in the TD-CFIE is restored by insertion of the appropriate Gram matrices in the TD-MFIE contribution. Numerical results confirm the necessity of this operation.

The scheme introduced here can easily be incorporated in existing MoT solvers, because the required integrations are equivalent to those employed in collocation scheme. For the same reason, the scheme is amenable to acceleration by the PWTD algorithm [10] or the TD-AIM [11].

This paper is organized as follows. In Section II, the standard Galerkin-in-space / collocation-in-time discretization of the TD-CFIE is revisited, and its shortcomings are highlighted.

In Section III, the temporal mixed Galerkin discretization is introduced. It is based on a first order temporal Galerkin scheme that was proven to be both stable and convergent for acoustic RP-BIEs [12] and for the TD-EFIE [13]. While this first order scheme is already more accurate than collocationin-time schemes, its accuracy is still limited by the first order expansion in time. Higher order Galerkin-in-time schemes can be employed to increase the accuracy for a given value of the time-step size. In Section III, a general order extension to the first order scheme which also maintains discrete causality is presented.

In Section IV, the spatial discretization is studied. Specifically, the non-conforming discretization of the TD-MFIE contribution, which deteriorates the accuracy of the TD-CFIE, is replaced by the conforming mixed discretization scheme proposed in [4]. Consistency with the TD-EFIE contribution is restored by insertion of the appropriate Gram matrices.

Finally, numerical results are presented in Section V, demonstrating the applicability of the proposed schemes, and providing insight into the gain of accuracy, both on smooth and non-smooth geometries.

\section{The Time Domain Combined Field Integral EQUATION}

Consider a PEC body occupying a domain $\Omega$ with boundary $\Gamma$ and exterior normal vector $\hat{\boldsymbol{n}}$. It resides in a background medium with permittivity $\epsilon$ and permeability $\mu$. Initially, the electromagnetic fields in the neighbourhood of $\Omega$ vanish. For $t>0$, an incident electromagnetic field $\boldsymbol{e}^{i}(\boldsymbol{r}, t), \boldsymbol{h}^{i}(\boldsymbol{r}, t)$ induces an unknown electric current $\boldsymbol{j}(\boldsymbol{r}, t)$ on $\Gamma$, which satisfies both the TD-EFIE and the TD-MFIE:

$$
\begin{aligned}
-\eta \mathcal{T} \boldsymbol{j}(\boldsymbol{r}, t) & =\hat{\boldsymbol{n}} \times \boldsymbol{e}^{i}(\boldsymbol{r}, t) \\
\left\{\frac{1}{2} \mathcal{I}+\mathcal{K}\right\} \boldsymbol{j}(\boldsymbol{r}, t) & =\hat{\boldsymbol{n}} \times \boldsymbol{h}^{i}(\boldsymbol{r}, t)
\end{aligned}
$$

$\forall t>0$ and $\forall \boldsymbol{r} \in \Gamma$, where

$$
\begin{aligned}
\mathcal{T} \boldsymbol{j}(\boldsymbol{r}, t) & =\mathcal{T}_{s} \boldsymbol{j}(\boldsymbol{r}, t)+\mathcal{T}_{h} \boldsymbol{j}(\boldsymbol{r}, t), \\
\mathcal{T}_{s} \boldsymbol{j}(\boldsymbol{r}, t) & =-\frac{1}{4 \pi c} \hat{\boldsymbol{n}} \times \int_{\Gamma} \frac{\partial_{t} \boldsymbol{j}\left(\boldsymbol{r}^{\prime}, t_{r}\right)}{R} d s^{\prime}, \\
\mathcal{T}_{h} \boldsymbol{j}(\boldsymbol{r}, t) & =\frac{c}{4 \pi} \hat{\boldsymbol{n}} \times p \cdot v \cdot \int_{\Gamma} \nabla \frac{\int_{0}^{t_{r}} \nabla^{\prime} \cdot \boldsymbol{j}\left(\boldsymbol{r}^{\prime}, t^{\prime}\right) d t^{\prime}}{R} d s^{\prime}, \\
\mathcal{K} \boldsymbol{j}(\boldsymbol{r}, t) & =-\frac{1}{4 \pi} \hat{\boldsymbol{n}} \times p \cdot v \cdot \int_{\Gamma} \nabla \times \frac{\boldsymbol{j}\left(\boldsymbol{r}^{\prime}, t_{r}\right)}{R} d s^{\prime},
\end{aligned}
$$

$\mathcal{I}$ is the identity operator, $c=(\epsilon \mu)^{-1 / 2}$ is the speed of light in the background medium, $R=\left|\boldsymbol{r}-\boldsymbol{r}^{\prime}\right|$ and $t_{r}=t-R / c$.

The TD-EFIE (1) and the TD-MFIE (2) are, however, plagued by spurious resonances [14]. In order to obtain a resonance free equation, the TD-EFIE and the TD-MFIE are combined into the TD-CFIE as follows [15]:

$$
\begin{array}{r}
-\alpha \eta \hat{\boldsymbol{n}} \times \mathcal{T} \boldsymbol{j}(\boldsymbol{r}, t)+\eta(1-\alpha)\left\{\frac{1}{2} \mathcal{I}+\mathcal{K}\right\} \boldsymbol{j}(\boldsymbol{r}, t) \\
=\alpha \hat{\boldsymbol{n}} \times \hat{\boldsymbol{n}} \times \boldsymbol{e}^{i}(\boldsymbol{r}, t)+\eta(1-\alpha) \hat{\boldsymbol{n}} \times \boldsymbol{h}^{i}(\boldsymbol{r}, t),
\end{array}
$$

where $\alpha$ is a dimensionless weighting parameter ranging from 0 (pure TD-MFIE) to 1 (pure TD-EFIE).

The temporal integration in $\mathcal{T}_{h}$ complicates the numerical solution of the TD-CFIE, but is eliminated by differentiating the TD-CFIE with respect to time:

$$
\begin{array}{r}
-\alpha \eta \hat{\boldsymbol{n}} \times \dot{\mathcal{T}} \boldsymbol{j}(\boldsymbol{r}, t)+\eta(1-\alpha)\left\{\frac{1}{2} \dot{\mathcal{I}}+\dot{\mathcal{K}}\right\} \boldsymbol{j}(\boldsymbol{r}, t) \\
=\alpha \hat{\boldsymbol{n}} \times \hat{\boldsymbol{n}} \times \dot{\boldsymbol{e}}^{i}(\boldsymbol{r}, t)+\eta(1-\alpha) \hat{\boldsymbol{n}} \times \dot{\boldsymbol{h}}^{i}(\boldsymbol{r}, t)
\end{array}
$$

where the dot on the operators and fields represents temporal differentiation. This equation can easily be discretized by expanding the unknown current $\boldsymbol{j}(\boldsymbol{r}, t)$ in a set of $N_{S}$ spatial basis functions $\boldsymbol{f}_{i}(\boldsymbol{r})$, and a set of shifted temporal basis functions $T_{j}(t)=T(t-j \Delta t)$ :

$$
\boldsymbol{j}(\boldsymbol{r}, t)=\sum_{i=1}^{N_{S}} \sum_{j=1}^{\infty} \mathrm{x}_{i}^{(j)} \boldsymbol{f}_{i}(\boldsymbol{r}) T_{j}(t)
$$

Traditionally, $\boldsymbol{f}_{i}(\boldsymbol{r})$ are chosen to be the RWG basis functions [2], while $T_{j}(t)$ are shifted piecewise polynomial Lagrange interpolators of order $p$ (Fig. 1, top panels) [3]. Next, the expansion (5) is inserted into the TD-CFIE (4). This equation is then spatially tested with the RWG functions $f_{i}$, and evaluated at $N_{T}$ subsequent time steps $t=k \Delta t$, $k=1,2, \ldots, N_{T}$. Due to time translation symmetry, this results in the following system of equations:

$$
\mathbf{Z}^{(0)} \mathbf{x}^{(j)}=\mathbf{v}^{(j)}-\sum_{i=0}^{j-1} \mathbf{Z}^{(j-i)} \mathbf{x}^{(i)}
$$



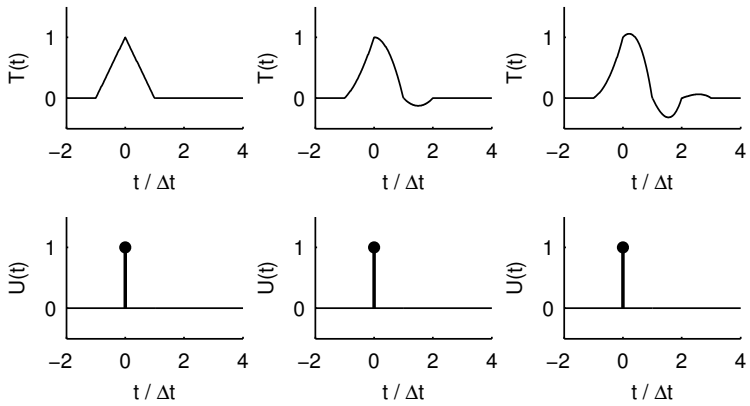

Fig. 1. Top: first (left), second (middle) and third (right) degree piecewise polynomial Lagrange interpolators. Bottom: the collocation-in-time method is equivalent to the space-time mixed Galerkin method with $U(t)=\delta(t)$.

where

$$
\begin{aligned}
\mathbf{v}_{m}^{(j)}= & -\alpha\left(\hat{\boldsymbol{n}} \times \boldsymbol{f}_{m}, \hat{\boldsymbol{n}} \times \dot{\boldsymbol{e}}^{i}\right)_{t=j \Delta t}+ \\
& \eta(1-\alpha)\left(\boldsymbol{f}_{m}, \hat{\boldsymbol{n}} \times \dot{\boldsymbol{h}}^{i}\right)_{t=j \Delta t}, \\
\mathbf{Z}_{m n}^{(j)}= & \alpha \eta\left(\hat{\boldsymbol{n}} \times \boldsymbol{f}_{m}, \dot{\mathcal{T}}\left[\boldsymbol{f}_{n} T\right]\right)_{t=j \Delta t}+ \\
& \eta(1-\alpha)\left(\boldsymbol{f}_{m},\left\{\frac{1}{2} \dot{\mathcal{I}}+\dot{\mathcal{K}}\right\}\left[\boldsymbol{f}_{n} T\right]\right)_{t=j \Delta t}, \\
(\boldsymbol{a}, \boldsymbol{b})_{t=j \Delta t}= & {\left[\int_{\Gamma} \boldsymbol{a}(\boldsymbol{r}, t) \cdot \boldsymbol{b}(\boldsymbol{r}, t) d s\right]_{t=j \Delta t} . }
\end{aligned}
$$

The system (6) is causal: for every time step $j$, the right hand side only depends on $\mathrm{x}^{(i)}, i<j$. It can therefore be solved successively for $\mathrm{x}^{(j)}, j=1,2,3, \ldots, N_{T}$, yielding the samples of the current $\boldsymbol{j}(\boldsymbol{r}, t)$ for $t \in\left[0, N_{T} \Delta t\right]$. This is the marching-on-in-time algorithm.

Different schemes exist for the numerical evaluation of the four dimensional integrals in $\mathbf{Z}^{(j)}$, see e.g. [16], [17], [18], [19], [20], [21]. In general, more accurate integration schemes give rise to algorithms that are stable for an increasingly broader range of geometries, material parameters, and discretization parameters.

The complexity of this MoT scheme scales as $\mathcal{O}\left(N_{T} N_{S}^{2}\right)$. However, this can be reduced to $\mathcal{O}\left(N_{T} N_{S}^{3 / 2} \log N_{S}\right)$ and even $\mathcal{O}\left(N_{T} N_{S} \log ^{2} N_{S}\right)$ using the two-level and multilevel plane wave time domain (PWTD) algorithms, respectively [10], or to $\mathcal{O}\left(N_{T} N_{S}^{3 / 2} \log ^{2} N_{S}\right)$ using the time domain adaptive integration method (TD-AIM) [11].

While the Galerkin-in-space / collocation-in-time scheme has been widely applied, its soundness remains questionable. First, the collocation-in-time scheme is not well understood. As it does not fit in the general finite element theoretical framework, classical coercivity arguments cannot be used to establish its stability. While convergence proofs have been formulated, they remain limited to specific collocation schemes applied to the scalar single layer potential equation for acoustic scattering [22], [23].

Furthermore, the spatial testing procedure is well understood from frequency domain applications (see e.g. [24]). It is known that the spatial discretization of the TD-MFIE employed in (6) is non-conforming and inaccurate [25], [6].
Finally, the expansion of the current in piecewise Lagrange interpolators of order $p$ limits the accuracy. Band-limited signals can be more accurately approximated using bandlimited interpolation functions (BLIFs, [26]) [27]. The resulting scheme is however not causal and requires an extrapolation procedure which introduces another source of errors.

\section{Temporal Mixed Galerkin Discretization of THE TD-CFIE}

\section{A. The Temporal Galerkin Method}

The concerns raised above motivate the search for another temporal discretization scheme for the TD-CFIE. The resulting system must be causal to allow for time-stepping, generalorder, and stable and convergent.

Consider an RP-BIE (such as the TD-CFIE) of the form

$$
\mathcal{O} \boldsymbol{j}(\boldsymbol{r}, t)=\boldsymbol{q}(\boldsymbol{r}, t),
$$

where the unknown current $\boldsymbol{j}(\boldsymbol{r}, t)$ is expanded as in (5). Eqn. (7) is now multiplied by a spatial testing function $\boldsymbol{f}_{m}(\boldsymbol{r})$ as well as a temporal testing function $U_{k}(t)=U(t-k \Delta t)$. The resulting equation is integrated over both space and time:

$$
\begin{array}{r}
\sum_{n, i} \mathrm{x}_{n}^{(i)} \int_{\Gamma \times \mathbb{R}} U_{k}(t) \boldsymbol{f}_{m}(\boldsymbol{r}) \cdot \mathcal{O}\left[\boldsymbol{f}_{n} T_{i}\right](\boldsymbol{r}, t) d s d t \\
=\int_{\Gamma \times \mathbb{R}} U_{k}(t) \boldsymbol{f}_{m}(\boldsymbol{r}) \cdot \boldsymbol{q}(\boldsymbol{r}, t) d s d t
\end{array}
$$

Due to time translation symmetry, this expression depends on $i$ and $k$ only through the difference $k-i$. Furthermore, if the support of $T(t)$ is bounded below, and the support of $U(t)$ is bounded above, the integrals in the left hand side of (8) vanish for sufficiently small values of $k-i$. Specifically, assume that $T(t)=0 \forall t<-\Delta t$ and $U(t)=0 \forall t>0$ (this can be accomplished by shifting the basis and testing functions, or shifting the temporal indices). Now the integrals with $k<i$ vanish, and the MoT equation becomes

$$
\mathbf{Z}^{(0)} \mathbf{x}^{(j)}=\mathbf{v}^{(j)}-\sum_{i=0}^{j-1} \mathbf{Z}^{(j-i)} \mathbf{x}^{(i)}
$$

where

$$
\begin{aligned}
\mathbf{v}_{m}^{(j)} & =\left(\boldsymbol{f}_{m} U_{j}, \boldsymbol{q}\right), \\
\mathbf{Z}_{m n}^{(j)} & =\left(\boldsymbol{f}_{m} U_{j}, \mathcal{O}\left[\boldsymbol{f}_{n} T\right]\right), \\
(\boldsymbol{a}, \boldsymbol{b}) & =\int_{\Gamma \times \mathbb{R}} \boldsymbol{a}(\boldsymbol{r}, t) \cdot \boldsymbol{b}(\boldsymbol{r}, t) d s d t .
\end{aligned}
$$

The collocation scheme described in the previous section can be recovered by setting $U(t)=\delta(t)$, the Dirac delta function (Fig. 1, bottom panels).

There is another relation between the interaction matrices $\mathbf{Z}^{(i)}$ of the temporal collocation and Galerkin schemes. By inverting the order of the temporal testing and the temporal convolution of the Green function with the temporal basis function, one obtains the equivalence between the matrix elements of a temporal Galerkin scheme with basis and testing functions

$$
T_{i}(t)=T(t-i \Delta t), \quad U_{i}(t)=U(t-i \Delta t)
$$


respectively, and those of a collocation scheme with basis functions

$$
\xi_{i}(t)=\int_{-\infty}^{\infty} U(\tau) T(t-i \Delta t+\tau) d \tau .
$$

This equivalence greatly simplifies the implementation of the space-time mixed Galerkin method in existing solvers, even when fast techniques or special integration routines are used. Note however that only the matrix elements are equal, whereas the excitation $v^{(j)}$ and the current expansion (5) are not. The stability only depends on the matrix elements. Thus, if the temporal Galerkin scheme is stable, the equivalent collocation scheme will also be stable. This has been exploited to construct a stable collocation scheme in [28].

Finally, it must be noted that the boundedness of the support of the basis and testing functions is not sufficient to obtain a usable scheme. For example, the BLIFs [26] of order M are defined on the interval $[-M \Delta t, M \Delta t]$. After shifting these basis functions to the interval $[-\Delta t,(2 M-1) \Delta t]$, one can obtain a causal scheme with a piecewise constant testing function that is one on the interval $[-\Delta t, 0]$ and zero everywhere else. However, the system matrix will contain interactions of the fields radiated by the first time segment in the BLIF's support, which are multiple orders of magnitude smaller than those radiated in the center segments of its support. This will result in large numerical errors leading to unstable results. This example illustrates that the temporal basis and testing functions must be carefully selected. The fields radiated by the basis function $T_{i}(t)$ must strongly overlap with the testing function $U_{i}(t)$ (in comparison to $U_{i+1}(t), U_{i+2}(t), \ldots$ ) in time, so that the matrix $\mathbf{Z}^{(0)}$ accounts for most of the near interactions. This can be achieved if both $T(t)$ and $U(t)$ are (approximately) localized in the interval $[-\Delta t, 0]$.

\section{B. First Order Basis and Testing Functions}

In [12], it is proven that a temporal Galerkin scheme, which makes use of first order basis functions and piecewise constant testing functions, yields a stable system when applied to the time-differentiated scalar first and second kind RP-BIEs for acoustic scattering, if the span of the testing functions is equal to the span of the temporal derivatives of the basis functions. Therefore, the author of [12] chose continuous, piecewise linear basis functions

$$
T(t)=\left\{\begin{array}{lr}
1+t / \Delta t & -\Delta t \leq t<0 \\
1-t / \Delta t & 0 \leq t \leq \Delta t \\
0 & \text { otherwise }
\end{array}\right.
$$

and piecewise constant testing functions

$$
U(t)=\left\{\begin{array}{rr}
1 & -\Delta t \leq t<0 \\
0 & \text { otherwise }
\end{array} .\right.
$$

$T(t)$ and $U(t)$ are shown in the left panels of Fig. 2. In [13], a similar first order in time scheme was applied to the TD-EFIE.

This scheme is only defined for lowest order approximations. As will be shown in the numerical results section, this limits the maximal achievable accuracy for a fixed time step $\Delta t$. A higher-order generalization is presented in the next section.


Fig. 2. Top: temporal current basis functions for first (left), second (middle) and third (right) order temporally mixed Galerkin scheme. Bottom: corresponding testing functions.

\section{Definition of Higher Order Temporal Basis and Testing Functions}

Now, in order to span all continuous, piecewise $p$-th order polynomial functions, introduce a set of $p$ basis functions $T^{(\mu)}(t), \mu=1, \ldots, p$ that are globally continuous, piecewise polynomials of order $p$, achieve the value one at $t=(\mu / p-$ 1) $\Delta t$, and zero at $t=(\nu / p-1) \Delta t, \nu \neq \mu$. The support of $T^{(\mu)}$ is chosen to be $[-\Delta t, \Delta t]$ for $\mu=p$, and $[-\Delta t, 0]$ for $\mu \neq p$. For example, for $p=2$ (Fig. 2, top middle panel):

$$
\begin{aligned}
& T^{(1)}(t)= \begin{cases}-4 \frac{t}{\Delta t}\left(\frac{t}{\Delta t}+1\right) & -\Delta t<t<0 \\
0 & \text { otherwise }\end{cases} \\
& T^{(2)}(t)= \begin{cases}2\left(\frac{t}{\Delta t}+1\right)\left(\frac{t}{\Delta t}+\frac{1}{2}\right) & -\Delta t<t<0 \\
2\left(\frac{t}{\Delta t}-1\right)\left(\frac{t}{\Delta t}-\frac{1}{2}\right) & 0 \leq t<\Delta t \\
0 & \text { otherwise }\end{cases}
\end{aligned}
$$

This introduces $p$ degrees of freedom per time step. The current expansion (5) then becomes

$$
\boldsymbol{j}(\boldsymbol{r}, t)=\sum_{i=1}^{N_{S}} \sum_{j=1}^{\infty} \sum_{\mu=1}^{p} \mathrm{x}_{i}^{(j, \mu)} \boldsymbol{f}_{i}(\boldsymbol{r}) T_{j}^{(\mu)}(t)
$$

Next, a set of $p$ testing functions $U^{(\mu)}(t), \mu=1, \ldots, p$ must be defined, that spans the space of the time derivatives of the basis functions. These can be chosen as the piecewise polynomials of order $p-1$ on the interval $[-\Delta t, 0]$, achieving the value one at $t=(\mu-p) /(p-1) \Delta t$, and zero on $t=(\nu-p) /(p-1) \Delta t$, $\nu \neq \mu$. For $p=2$ (Fig. 2, bottom middle panel):

$$
\begin{aligned}
U^{(1)}(t) & = \begin{cases}-\frac{t}{\Delta t} & -\Delta t<t<0 \\
0 & \text { otherwise }\end{cases} \\
U^{(2)}(t) & =\left\{\begin{array}{ll}
\frac{t}{\Delta t}+1 & -\Delta t<t<0 \\
0 & \text { otherwise }
\end{array} .\right.
\end{aligned}
$$

Note that the testing functions are not continuous at the boundary between adjacent time segments, whereas the basis functions are.

Since the higher order basis and testing functions associated to the same time step do not fulfil the discrete causality condition among each other, the MoT system needs to be solved simultaneously for the corresponding unknowns. In 
other words, the presence of higher order basis and testing functions introduces block structure in the system matrix. More explicitly, the marching-on-in-time equations become:

$$
\begin{gathered}
\left(\begin{array}{ccc}
\mathbf{Z}_{11}^{(0)} & \ldots & \mathbf{Z}_{1 p}^{(0)} \\
\ldots & \ldots & \ldots \\
\mathbf{Z}_{p 1}^{(0)} & \ldots & \mathbf{Z}_{p p}^{(0)}
\end{array}\right)\left(\begin{array}{c}
\mathbf{x}^{(j, 1)} \\
\ldots \\
\mathbf{x}^{(j, p)}
\end{array}\right) \\
=\left(\begin{array}{c}
\mathbf{v}^{(j, 1)} \\
\ldots \\
\mathbf{v}^{(j, p)}
\end{array}\right)-\sum_{i=0}^{j-1}\left(\begin{array}{ccc}
\mathbf{Z}_{11}^{(i)} & \ldots & \mathbf{Z}_{1 p}^{(i)} \\
\ldots & \ldots & \ldots \\
\mathbf{Z}_{p 1}^{(i)} & \ldots & \mathbf{Z}_{p p}^{(i)}
\end{array}\right)\left(\begin{array}{c}
\mathbf{x}^{(j-i, 1)} \\
\ldots \\
\mathbf{x}^{(j-i, p)}
\end{array}\right) \\
\mathbf{v}_{m}^{(j, \mu)}=\left(\boldsymbol{f}_{m} U_{j}^{(\mu)}, \boldsymbol{q}\right) \\
\left(\mathbf{Z}_{\mu \nu}^{(j)}\right)_{m n}=\left(\boldsymbol{f}_{m} U_{j}^{(\mu)}, \mathcal{O}\left[\boldsymbol{f}_{n} T^{(\nu)}(t)\right]\right) .
\end{gathered}
$$

$\mathbf{Z}_{\mu \nu}^{(j)}, \quad \mu, \nu=1,2, \ldots, p$ are block matrices of dimension $N_{S} \times N_{S}$. Thus, $p N_{S}$ unknowns are treated at each time step, increasing the order of the system to be inverted at every time step with a factor $p$. Note, however, that this is in no way different from the increase of non zero matrix entries associated with an increase in order in e.g. classic finite element methods.

\section{Mixed Spatial Discretization of the TD-CFIE}

It is well known that the frequency domain MFIE discretized using RWG basis and testing functions, yields inaccurate results [4], [25]. The spatial discretization of the TD-CFIE used in Section II,

$$
\begin{aligned}
& \eta \alpha(\hat{\boldsymbol{n}} \times \boldsymbol{f}, \dot{\mathcal{T}} \boldsymbol{j})+\eta(1-\alpha)\left(\boldsymbol{f},\left\{\frac{1}{2} \dot{\mathcal{I}}+\dot{\mathcal{K}}\right\} \boldsymbol{j}\right) \\
= & -\alpha\left(\hat{\boldsymbol{n}} \times \boldsymbol{f}, \hat{\boldsymbol{n}} \times \dot{\boldsymbol{e}}^{i}\right)+\eta(1-\alpha)\left(\boldsymbol{f}, \hat{\boldsymbol{n}} \times \dot{\boldsymbol{h}}^{i}\right),
\end{aligned}
$$

incorporates the time domain version of this non-conforming discretization scheme for the MFIE contribution. In the numerical results section, it will be illustrated that this nonconforming scheme indeed deteriorates the accuracy of the results. A similar phenomenon has been observed in the frequency domain CFIE [6].

In [4] a mixed discretization scheme using RWG functions as basis functions and $\mathrm{BC}$ functions as testing functions for the frequency domain MFIE has been introduced. This scheme is conforming and leads to well-conditioned system matrices. The accuracy of this scheme's solution has been shown to be competitive with that of the EFIE [4], [29].

The accuracy of the TD-CFIE can thus be improved by incorporating this conforming discretization scheme for the MFIE. By testing the TD-EFIE using RWG functions, testing the TD-MFIE using the rotated BC functions $\hat{\boldsymbol{n}} \times \boldsymbol{g}_{m}$, and adding both contributions, one obtains:

$$
\begin{aligned}
& \eta \alpha(\hat{\boldsymbol{n}} \times \boldsymbol{f}, \dot{\mathcal{T}} \boldsymbol{j})+\eta(1-\alpha)\left(\hat{\boldsymbol{n}} \times \boldsymbol{g},\left\{\frac{1}{2} \dot{\mathcal{I}}+\dot{\mathcal{K}}\right\} \boldsymbol{j}\right) \\
& =-\alpha\left(\hat{\boldsymbol{n}} \times \boldsymbol{f}, \hat{\boldsymbol{n}} \times \dot{\boldsymbol{e}}^{i}\right)+\eta(1-\alpha)\left(\hat{\boldsymbol{n}} \times \boldsymbol{g}, \hat{\boldsymbol{n}} \times \dot{\boldsymbol{h}}^{i}\right)
\end{aligned}
$$

A frequency domain version of this discretization scheme has been suggested in [5]. Although a significant increase in accuracy was reported, this is not a consistent discretization of the CFIE, since the EFIE and MFIE contributions are tested using different functions. In the numerical results section, it will be shown that it is still prone to spurious resonances.

A consistent conforming discretization of the frequency domain CFIE was obtained in [9] by applying the Calderón multiplicative preconditioner to the EFIE contribution. A mixed discretization was applied to the Calderón preconditioned EFIE (CP-EFIE), such that both the CP-EFIE and the MFIE are tested using BC functions. However, for geometries discretized with moderately dense meshes, the introduction of a Calderón preconditioner introduces unnecessary overhead.

A consistent and resonance free mixed discretization of the TD-CFIE (4) can be obtained without applying a Calderón multiplicative preconditioner, by projecting the range of the TD-MFIE contribution onto the RWG basis:

$$
\left\{\frac{1}{2} \dot{\mathcal{I}}+\dot{\mathcal{K}}\right\} \boldsymbol{j}(\boldsymbol{r}, t) \approx \sum_{m} \mathrm{~b}_{m}(t) \boldsymbol{f}_{m}(\boldsymbol{r}) \quad \forall t .
$$

This equation can be solved for $\mathrm{b}(t)$ by spatially testing it with the rotated BC functions $\hat{\boldsymbol{n}} \times \boldsymbol{g}_{n}(\boldsymbol{r})$. In matrix form:

$$
\mathrm{b}(t)=\mathbf{G}_{\hat{\boldsymbol{n}} \times \boldsymbol{g}, \boldsymbol{f}}^{-1} \cdot\left(\hat{\boldsymbol{n}} \times \boldsymbol{g},\left\{\frac{1}{2} \dot{\mathcal{I}}+\dot{\mathcal{K}}\right\} \boldsymbol{j}(t)\right),
$$

where the Gram matrix $\mathbf{G}_{\hat{\boldsymbol{n}} \times \boldsymbol{g}, \boldsymbol{f}}$ is given by

$$
\left(\mathbf{G}_{\hat{\boldsymbol{n}} \times \boldsymbol{g}, \boldsymbol{f}}\right)_{m n}=\left(\hat{\boldsymbol{n}} \times \boldsymbol{g}_{m}, \boldsymbol{f}_{n}\right) .
$$

The expansion (20) is inserted into the TD-CFIE (4). A similar projection is applied to the incoming magnetic field $\boldsymbol{h}^{i}(\boldsymbol{r}, t)$. Finally, the resulting equation is tested with the RWG functions $\boldsymbol{f}_{m}(\boldsymbol{r})$. This results in

$$
\begin{aligned}
& \eta \alpha(\hat{\boldsymbol{n}} \times \boldsymbol{f}, \dot{\mathcal{T}} \boldsymbol{j}) \\
& +\eta(1-\alpha) \mathbf{G}_{\boldsymbol{f}, \boldsymbol{f}} \mathbf{G}_{\hat{\boldsymbol{n}} \times \boldsymbol{g}, \boldsymbol{f}}^{-1}\left(\hat{\boldsymbol{n}} \times \boldsymbol{g},\left\{\frac{1}{2} \dot{\mathcal{I}}+\dot{\mathcal{K}}\right\} \boldsymbol{j}\right) \\
& =-\alpha\left(\hat{\boldsymbol{n}} \times \boldsymbol{f}, \hat{\boldsymbol{n}} \times \dot{\boldsymbol{e}}^{i}\right) \\
& +\eta(1-\alpha) \mathbf{G}_{\boldsymbol{f}, \boldsymbol{f}} \mathbf{G}_{\hat{\boldsymbol{n}} \times \boldsymbol{g}, \boldsymbol{f}}^{-1}\left(\hat{\boldsymbol{n}} \times \boldsymbol{g}, \hat{\boldsymbol{n}} \times \dot{\boldsymbol{h}}^{i}\right)
\end{aligned}
$$

where the Gram matrix $\mathbf{G}_{\boldsymbol{f}, \boldsymbol{f}}$ results from testing the expansion (20) with the RWG functions $\boldsymbol{f}_{m}(\boldsymbol{r})$ :

$$
\left(\mathbf{G}_{\boldsymbol{f}, \boldsymbol{f}}\right)_{m n}=\left(\boldsymbol{f}_{m}, \boldsymbol{f}_{n}\right) .
$$

(22) is a combination of spatially conforming discretizations of the TD-EFIE and the TD-MFIE, but also a consistent spatial discretization of the TD-CFIE as a whole (4). Finally, the full discretization is achieved by applying either the classical collocation-in-time scheme or the temporal mixed Galerkin scheme introduced above.

Alternatively, the range of the TD-EFIE operator can be projected onto the $\mathrm{BC}$ basis:

$$
\dot{\mathcal{T}} \boldsymbol{j}(\boldsymbol{r}, t) \approx \sum_{m} \mathrm{c}_{m}(t) \boldsymbol{g}_{m}(\boldsymbol{r}) .
$$

This equation can be solved for $\mathrm{c}(t)$ by spatially testing it with the rotated RWG functions $\hat{\boldsymbol{n}} \times \boldsymbol{f}_{m}(\boldsymbol{r})$ :

$$
\mathrm{c}(t)=\mathbf{G}_{\hat{\boldsymbol{n}} \times \boldsymbol{f}, \boldsymbol{g}}^{-1} \cdot(\hat{\boldsymbol{n}} \times \boldsymbol{f}, \dot{\mathcal{T}} \boldsymbol{j}(t)),
$$


where the Gram matrix $\mathbf{G}_{\hat{\boldsymbol{n}} \times \boldsymbol{f}, \boldsymbol{g}}$ is given by

$$
\left(\mathbf{G}_{\hat{\boldsymbol{n}} \times \boldsymbol{f}, \boldsymbol{g}}\right)_{m n}=\left(\hat{\boldsymbol{n}} \times \boldsymbol{f}_{m}, \boldsymbol{g}_{n}\right) .
$$

The expansion (24) is inserted into the TD-CFIE (4). A similar projection is applied to the incoming electric field $\boldsymbol{e}^{i}(\boldsymbol{r}, t)$. Finally, the equation is tested with the BC functions $\boldsymbol{g}_{m}(\boldsymbol{r})$. This results in

$$
\begin{aligned}
& \eta \alpha \mathbf{G}_{\boldsymbol{g}, \boldsymbol{g}} \mathbf{G}_{\hat{\boldsymbol{n}} \times \boldsymbol{f}, \boldsymbol{g}}^{-1}(\hat{\boldsymbol{n}} \times \boldsymbol{f}, \dot{\mathcal{T}} \boldsymbol{j}) \\
& +\eta(1-\alpha)\left(\hat{\boldsymbol{n}} \times \boldsymbol{g},\left\{\frac{1}{2} \dot{\mathcal{I}}+\dot{\mathcal{K}}\right\} \boldsymbol{j}\right) \\
& =\quad-\alpha \mathbf{G}_{\boldsymbol{g}, \boldsymbol{g}} \mathbf{G}_{\hat{\boldsymbol{n}} \times \boldsymbol{f}, \boldsymbol{g}}^{-1}\left(\hat{\boldsymbol{n}} \times \boldsymbol{f}, \hat{\boldsymbol{n}} \times \dot{\boldsymbol{e}}^{i}\right) \\
& +\eta(1-\alpha)\left(\hat{\boldsymbol{n}} \times \boldsymbol{g}, \hat{\boldsymbol{n}} \times \dot{\boldsymbol{h}}^{i}\right),
\end{aligned}
$$

where the Gram matrix $\mathbf{G}_{\boldsymbol{g}, \boldsymbol{g}}$ results from testing the expansion (24) with the BC functions $\boldsymbol{g}_{m}(\boldsymbol{r})$ :

$$
\left(\mathbf{G}_{\boldsymbol{g}, \boldsymbol{g}}\right)_{m n}=\left(\boldsymbol{g}_{m}, \boldsymbol{g}_{n}\right) \text {. }
$$

Both (22) and (27) have been implemented and tested, but no significant difference in performance has been found. In the next parts, the first version (22) will be used.

The projection operators in (22) and (27) have been introduced ad hoc, i.e., to allow for the inclusion of the conforming discretization of the MFIE contribution. Concatenation of the MFIE operator with another operator, as in (22), has however been used to develop a rigorous variational analysis of the CFIE, applicable to non-smooth surfaces [30]. Concatenation of the EFIE operator with another operator, as in (27), has been used to improve the spectral properties of the CFIE operator [9], [31].

\section{NUMERICAL EXAMPLES}

In this section, numerical results demonstrating the accuracy and stability of the scheme introduced in the previous sections will be presented. First, the error estimation scheme used will detailed. To clearly indicate that both the spatial and temporal compartments of the scheme are needed in order to obtain accurate solutions, results will be shown obtained using all four combinations of temporal and spatial discretization schemes.

\section{A. Error Estimation Scheme and Rationale}

In order to assess the accuracy of an MoT solution, it will be compared to a known frequency domain reference solution $\boldsymbol{j}_{\text {ref }}(\boldsymbol{r}, \omega)$. In all the examples presented here, the scatterer is illuminated by a Gaussian pulse:

$$
\begin{aligned}
& \boldsymbol{e}^{i}(\boldsymbol{r}, t) \\
= & \frac{4 A}{w \sqrt{\pi}} \hat{\boldsymbol{p}} \exp \left(-\left(\frac{4}{w}\left(c\left(t-t_{0}\right)-\hat{\boldsymbol{k}} \cdot \boldsymbol{r}\right)\right)^{2}\right) \\
= & \frac{A \hat{\boldsymbol{p}}}{2 \pi c} \int_{-\infty}^{\infty} d \omega \exp \left(j \omega\left(t-t_{0}\right)-j \frac{\omega}{c} \hat{\boldsymbol{k}} \cdot \boldsymbol{r}-\left(\frac{\omega w}{8 c}\right)^{2}\right),
\end{aligned}
$$

where $A$ is the amplitude, $w$ is the width of the pulse, $\hat{\boldsymbol{p}}$ is the polarization vector, $c$ is the speed of light in vacuum, $\hat{k}$ is the direction in which the pulse is propagating, and $t_{0}$ is the time of arrival of the pulse at $\boldsymbol{r}=\mathbf{0}$. It can be interpreted as a superposition of plane waves with angular frequency $\omega$ and amplitude $\frac{A}{2 \pi c} \exp \left(-\left(\frac{\omega w}{8 c}\right)^{2}\right)$.

The current induced on a PEC surface by this incident wave is then computed using the MoT algorithm. To obtain the frequency response $\boldsymbol{j}_{\mathrm{MoT}}(\boldsymbol{r}, \omega)$, the current is Fourier transformed, and divided by $\frac{A}{c} \cdot \exp \left(-j \omega t_{0}-\left(\frac{\omega w}{8 c}\right)^{2}\right)$.

Now, $j_{\mathrm{MoT}}(\boldsymbol{r}, \omega)$ can be compared with the reference solution $\boldsymbol{j}_{\text {ref }}(\boldsymbol{r}, \omega)$. An often used measure for the error is the maximum error or the RMS error in the radar cross section. However, this does not account for errors in the near field. Here, another error measure is chosen: the $H_{\mathrm{div}}^{-1 / 2}$ norm on $\Gamma$. Since the operator

$$
\mathcal{S} \boldsymbol{j}(\boldsymbol{r}, \omega)=\frac{\omega}{c} \int_{\Gamma} d s^{\prime} \frac{\boldsymbol{j}\left(\boldsymbol{r}^{\prime}, \omega\right)}{\left|\boldsymbol{r}-\boldsymbol{r}^{\prime}\right|}-\frac{c}{\omega} \nabla \int_{\Gamma} d s^{\prime} \frac{\nabla^{\prime} \cdot \boldsymbol{j}\left(\boldsymbol{r}^{\prime}, \omega\right)}{\left|\boldsymbol{r}-\boldsymbol{r}^{\prime}\right|}
$$

is self-adjoint, positive, continuous and coercive with regard to the standard Sobolev-Slobodeckij norm on $H_{\mathrm{div}}^{-1 / 2}$, it furnishes a norm on $H_{\text {div }}^{-1 / 2}$. As follows from the trace theorems for electromagnetic fields [32], this norm is a measure for the near field energy of the error. Here, the discretization of $\mathcal{S}$ with respect to the triangle mesh is used to estimate the error in near field energy.

For each frequency $f=\omega / 2 \pi$, the relative error is defined as:

$$
\begin{aligned}
e_{r}(\omega)= & \left\|\boldsymbol{j}_{\mathrm{MoT}}-\boldsymbol{j}_{\mathrm{ref}}\right\|_{H_{\mathrm{div}}^{-1 / 2}} /\left\|\boldsymbol{j}_{\mathrm{ref}}\right\|_{H_{\mathrm{div}}^{-1 / 2}} \\
\|\boldsymbol{j}\|_{H_{\mathrm{div}}^{-1 / 2}}^{2}= & \frac{c}{\omega} \int_{\Gamma} d s \int_{\Gamma} d s^{\prime} \frac{\nabla \cdot \boldsymbol{j}(\boldsymbol{r}, \omega) \nabla^{\prime} \cdot \boldsymbol{j}\left(\boldsymbol{r}^{\prime}, \omega\right)}{\left|\boldsymbol{r}-\boldsymbol{r}^{\prime}\right|} \\
& +\frac{\omega}{c} \int_{\Gamma} d s \int_{\Gamma} d s^{\prime} \frac{\boldsymbol{j}(\boldsymbol{r}, \omega) \cdot \boldsymbol{j}\left(\boldsymbol{r}^{\prime}, \omega\right)}{\left|\boldsymbol{r}-\boldsymbol{r}^{\prime}\right|}
\end{aligned}
$$

This provides a physically meaningful estimate of the quality of a MoT solution.

For spherical scatterers, the solution can be computed analytically using the Mie series [33]. As the Mie series is defined on the sphere, whereas the MoT solution is defined on the triangle mesh, the projection of the Mie series $j_{\text {Mie }}(\boldsymbol{r}, \omega)$ onto the RWG basis is needed:

$$
\tilde{\boldsymbol{j}}_{\mathrm{Mie}}(\boldsymbol{r}, \omega)=\sum_{i} \mathrm{a}_{i}(\omega) \boldsymbol{f}_{i}(\boldsymbol{r}) .
$$

The expansion coefficients $\mathrm{a}_{i}$ are found by testing with rotated BC functions $\hat{\boldsymbol{n}} \times \boldsymbol{g}_{m}$, in order to conformingly discretize the unit operator [5]:

$$
\mathrm{a}(\omega)=\left(G_{\hat{\boldsymbol{n}} \times \boldsymbol{g}, \boldsymbol{f}}\right)^{-1}\left(\hat{\boldsymbol{n}} \times \boldsymbol{g}, \boldsymbol{j}_{\mathrm{Mie}}(\boldsymbol{r}, \omega)\right) .
$$

This projection is used as the reference solution in Section V-B.

For non-spherical scatterers, for which no analytical solution is available, one can instead use a simulation, of which the reliability is already established, as a reference solution. In Sections V-C and V-D, frequency domain EFIE simulations will be used for this purpose. The frequency domain EFIE is known to be reliable away from resonant frequencies. 


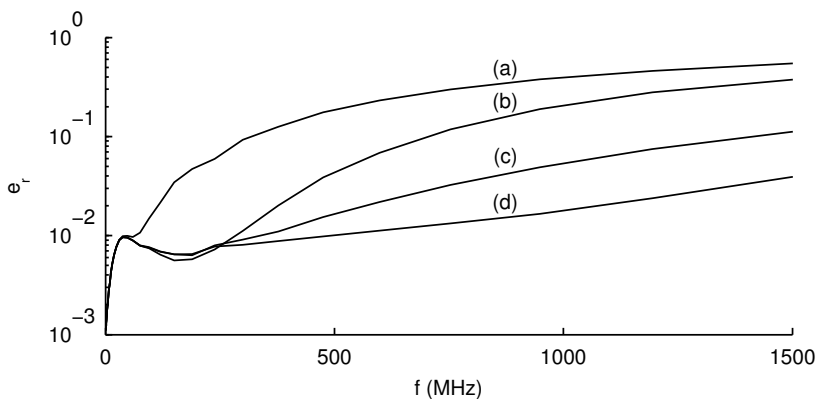

Fig. 3. Relative $H_{\text {div }}^{-1 / 2}$ error for different temporal discretizations: (a) collocation-in-time using second degree Lagrange interpolators, (b) collocation-in-time using third degree Lagrange interpolators, (c) first order temporal mixed Galerkin, and (d) second order temporal mixed Galerkin.

\section{B. Scattering by a Sphere}

Consider a PEC sphere of radius 0.3 meters, approximated using a equilateral triangle mesh with edge length $4.5 \mathrm{~cm}$. It is illuminated by a Gaussian pulse (with $w=3.33 \mathrm{~ns}, t_{0}=20$ $\mathrm{ns}$ ). The induced current is approximated by an expansion in 1887 RWG basis functions. The CFIE weighting coefficient is $\alpha=0.5$, unless specified otherwise.

1) Temporal Discretization Scheme: First, the temporal discretization scheme is investigated. In Fig. 3, the following schemes are compared:

(a) collocation-in-time using second degree Lagrange interpolators $(\Delta t=0.1 \mathrm{~ns})$,

(b) collocation-in-time using third degree Lagrange interpolators $(\Delta t=0.1 \mathrm{~ns})$,

(c) first order temporal mixed Galerkin $(\Delta t=0.1 \mathrm{~ns})$,

(d) second order temporal mixed Galerkin $(\Delta t=0.2 \mathrm{~ns})$.

The spatial discretization is performed using the mixed scheme (22). In (d), the time step is doubled, as there are $p=2$ degrees of freedom at each time step. Thus, simulations with an equal number of temporal degrees of freedom per unit of time are compared.

The $H_{\mathrm{div}}^{-1 / 2}$ error on the currents with respect to the Mie series are computed, as outlined in the previous section (Fig. 3). Temporal mixed Galerkin schemes outperform collocation schemes using an equal number of temporal degrees of freedom per unit of time. Using higher order temporal basis functions increases the accuracy even further.

In addition to the improved accuracy, the second order temporal Galerkin method exhibits faster convergence than the other schemes when the time step is decreased. In Fig. 4, the relative error as a function of $\Delta t / p$ (the inverse of the number of degrees of freedom per unit of time) at a fixed frequency of $500 \mathrm{MHz}$ is shown for the four temporal discretization types under investigation; all four types of temporal discretization make use of the mixed discretization scheme in space. Indeed, the second order temporal mixed Galerkin method exhibits faster convergence (approx. $\mathcal{O}\left(\Delta t^{4}\right)$ ) than both the first order Galerkin scheme and the second and third degree collocation schemes (approx. $\mathcal{O}\left(\Delta t^{2}\right), \mathcal{O}\left(\Delta t^{1}\right)$ and $\mathcal{O}\left(\Delta t^{2}\right)$, respectively). For small time steps, the error saturates toward a value determined by the density of the triangle mesh.

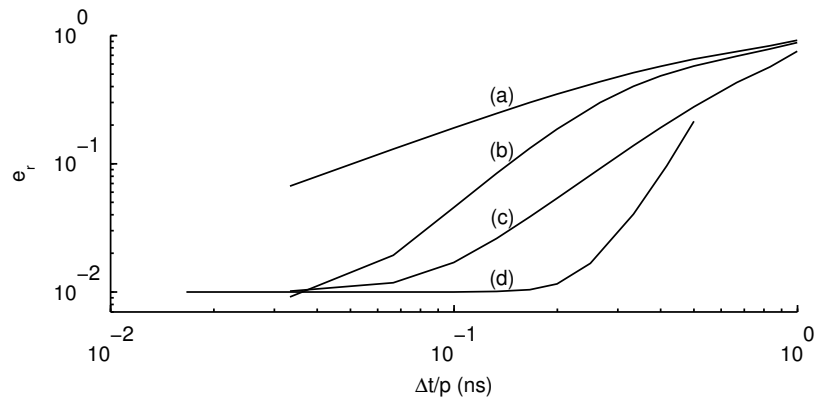

Fig. 4. The relative error at $500 \mathrm{MHz}$, for varying time step $\Delta t$ : (a) collocation-in-time using second degree Lagrange interpolators, (b) collocation-in-time using third degree Lagrange interpolators, (c) first order temporal mixed Galerkin, and (d) second order temporal mixed Galerkin.
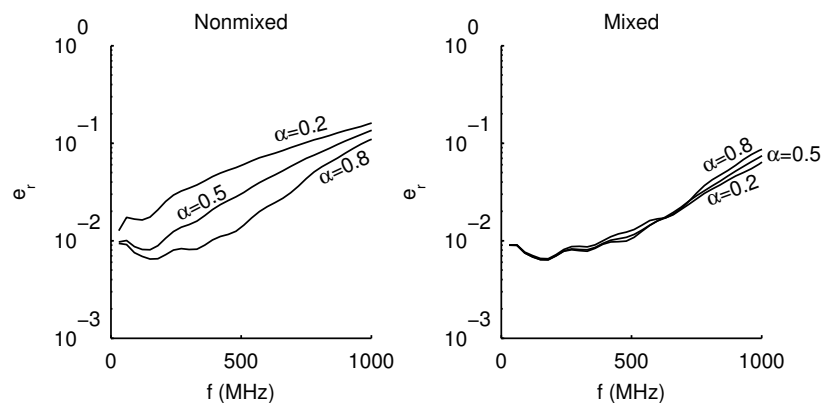

Fig. 5. The relative error for $\alpha=0.2, \alpha=0.5$ and $\alpha=0.8$. Left: non-mixed spatial discretization; right: mixed spatial discretization.

2) Spatial Discretization Scheme: In Section IV, it was claimed that the non-conforming discretization of the TDMFIE is responsible for the loss of accuracy, but that it can be remedied by the mixed spatial discretization. This becomes apparent when the errors are plotted for different values of the TD-CFIE weighting parameter $\alpha$. This is done in Fig. 5 for a second order temporal mixed Galerkin simulation with $\Delta t=0.4$ ns. For a spatial non-mixed discretization (left panel), the simulation becomes more accurate as $\alpha$ is increased, because the TD-MFIE contribution becomes smaller. For a spatial mixed discretization (right panel), the value of $\alpha$ does not significantly affect the accuracy. The complete range of values for $\alpha$ thus becomes available, whereas classic schemes are subject to a stability/accuracy trade-off.

Next, it is demonstrated that the restoration of consistency between the EFIE and MFIE contributions by multiplication with the appropriate Gram matrices is essential to arrive at a resonance-free scheme. Results of the following schemes are compared:

(1) the consistent non-mixed discretization (18),

(2) the inconsistent mixed discretization (19),

(3) the consistent mixed discretization (22).

The temporal discretization is performed using a first order temporal mixed Galerkin scheme with $\Delta t=0.1 \mathrm{~ns}$. The relative $H_{\text {div }}^{-1 / 2}$ error is plotted in Fig 6 .

The consistent schemes (1) and (3) give rise to smooth error curves, whereas the inconsistent scheme (2) picks up the resonant frequencies of the sphere. Indeed, the resonant 


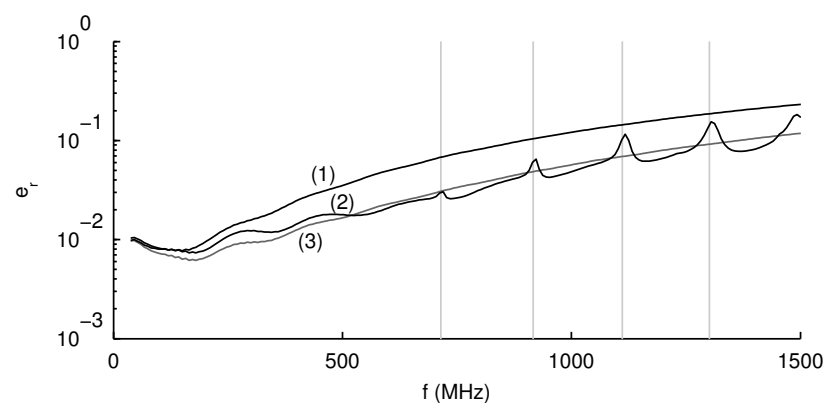

Fig. 6. The relative error for (1) the consistent non-mixed discretization (18), (2) the inconsistent mixed discretization (19), and (3), the consistent mixed discretization (22). The error in (2) shows distinct peaks at the resonance frequencies of the sphere, which are denoted by the vertical lines.

frequencies of a sphere with radius $r$ are determined by [34]:

$$
\begin{array}{ll}
J_{n+1 / 2}\left(\frac{2 \pi f r}{c}\right)=0 & \text { (TE modes), } \\
J_{n+1 / 2}^{\prime}\left(\frac{2 \pi f r}{c}\right)=0 & \text { (TM modes), }
\end{array}
$$

where $n$ is an integer, $J_{n}$ is the Bessel function of order $n$, and $J_{n}^{\prime}$ is its derivative. The peaks are found at the TE resonances at $715 \mathrm{MHz}(n=1), 917 \mathrm{MHz}(n=2), 1111 \mathrm{MHz}(n=3)$ and $1301 \mathrm{MHz}(n=4)$, which are represented as vertical lines in Fig. 6.

Away from these resonant frequencies, the mixed schemes (2) and (3) both are more accurate than the nonmixed scheme (1). In the neighbourhood of the resonant frequencies, the increased accuracy of (2) is partly lost due to the presence of spurious resonances. Therefore, an accurate solution over a broad frequency band can only be obtained using the consistent mixed scheme (3).

3) Space-Time Mixed Scheme: The importance of using the mixed spatial discretization scheme in conjunction with the higher order mixed temporal discretization scheme, is illustrated in Fig. 7. Here, the following simulations are compared:

(a) collocation-in-time using third degree Lagrange interpolators ( $\Delta t=0.2 \mathrm{~ns}$ ), nonmixed spatial discretization,

(b) collocation-in-time using third degree Lagrange interpolators $(\Delta t=0.2 \mathrm{~ns})$, mixed spatial discretization,

(c) second order Galerkin-in-time $(\Delta t=0.4 \mathrm{~ns})$, nonmixed spatial discretization,

(d) second order Galerkin-in-time $(\Delta t=0.4 \mathrm{~ns})$, mixed spatial discretization.

At low frequencies, the error is dominated by the spatial discretization scheme. At high frequencies, the error is dominated by the temporal discretization scheme. To achieve a high accuracy over a broad frequency band, the space-time mixed Galerkin scheme is needed.

\section{Scattering by a Cuboid}

As a second example, consider a PEC cuboid with dimensions 0.25 by 1 by 0.5 meters (Fig. 8). It is illuminated by a Gaussian pulse propagating along the positive $\mathrm{z}$-axis, with the

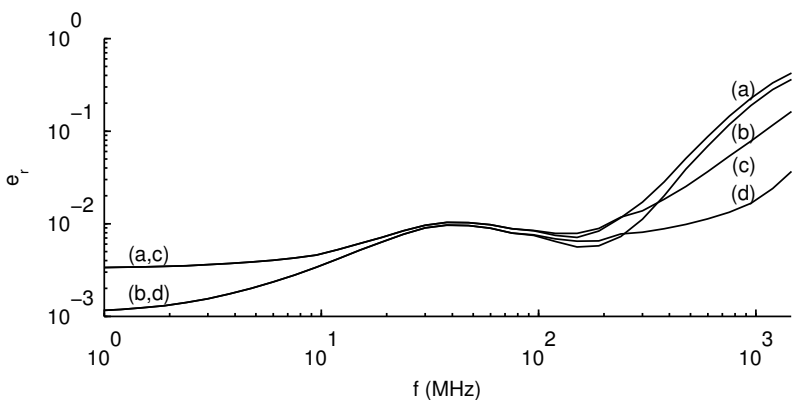

Fig. 7. The relative error for (a) collocation-in-time using third degree Lagrange interpolators and nonmixed spatial discretization, (b) collocation-intime using third degree Lagrange interpolators and mixed spatial discretization, (c) second order Galerkin-in-time and nonmixed spatial discretization, and (d) second order Galerkin-in-time and mixed spatial discretization.

electric field linearly polarized along the $\mathrm{x}$-axis. The induced current is approximated by an expansion in 1344 RWG basis functions. This scattering problem is simulated in the time domain using the following MoT schemes:

(a) spatial non-mixed collocation-in-time using second degree Lagrange interpolators $(\Delta t=0.1 \mathrm{~ns})$,

(b) spatial mixed collocation-in-time using second degree Lagrange interpolators $(\Delta t=0.1 \mathrm{~ns})$,

(c) spatial non-mixed second order temporal Galerkin $(\Delta t=0.2 \mathrm{~ns})$, and

(d) second order space-time mixed Galerkin ( $\Delta t=0.2 \mathrm{~ns})$.

As no analytical solution is available, frequency domain EFIE simulations are performed for frequencies ranging from 30 to $1000 \mathrm{MHz}$, with steps of $30 \mathrm{MHz}$, and used as a reference solution. The relative $H_{d i v}^{-1 / 2}$ error $e_{r}$ is computed for each of these frequencies (Fig. 9). For a number of discrete frequencies, the error peaks. This is due to the spurious resonances that plague the frequency domain EFIE. Indeed, the resonance frequencies of a cuboid with dimensions $x \times y \times z$ are [33]:

$$
f_{m n l}=c \sqrt{\left(\frac{m}{2 x}\right)^{2}+\left(\frac{n}{2 y}\right)^{2}+\left(\frac{l}{2 z}\right)^{2}}
$$

where $m, n$ and $l$ are integers. The largest peaks are located at 540 and $750 \mathrm{MHz}$, which are very close to the resonant frequencies of 540.46 and $749.48 \mathrm{MHz}$, respectively. Two small peaks are located at 420 and $630 \mathrm{MHz}$, which are due to the resonances at 423.97 and $618.04 \mathrm{MHz}$, respectively.

For low frequencies $(f<200 \mathrm{MHz}$ ), the error is dominated by the spatial discretization. In this frequency range, the mixed spatial discretization schemes are more accurate than the nonmixed ones. For higher frequencies, the error is dominated by the temporal discretization, and the temporal mixed Galerkin schemes are more accurate than the collocation schemes. Over the whole frequency range, the space-time mixed Galerkin scheme yields the most accurate results.

These results further support the conclusions drawn in Sections III and IV: even for non-smooth geometries, the spacetime mixed Galerkin scheme is significantly more accurate than the tradititial non-mixed collocation scheme. 


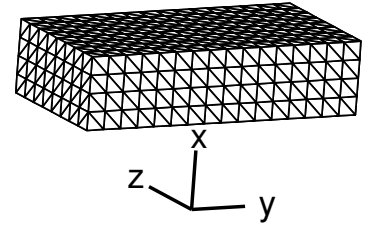

Fig. 8. A cuboid with dimensions 0.25 by 1 by 0.5 meters, discretized using 896 right triangles. The incident electromagnetic field is propagating in the $\mathrm{z}$-direction, while the electric field is polarized along the x-axis.

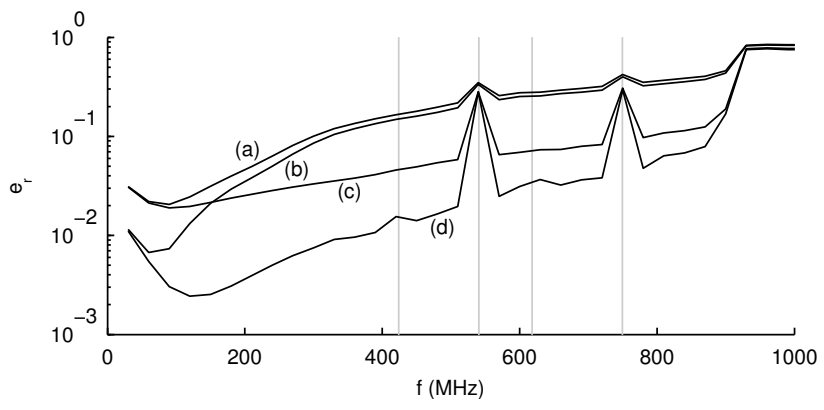

Fig. 9. Relative $H_{\text {div }}^{-1 / 2}$ error of the TD-CFIE simulations for scattering by a cuboid with respect to the frequency domain EFIE. The error peaks at the resonance frequencies of the cuboid, indicated by the vertical lines.

\section{Scattering by an Ice Cream Cone}

As a third example, scattering by the PEC ice cream cone depicted in Fig. 10 is simulated. Due to the sharp point, the geometry is even more singular than the cuboid. Again, the electric field is polarized along the $\mathrm{x}$-axis, while the wave propagates along the z-axis. The current is approximated by an expansion in 1845 RWG basis functions.

The following time domain simulations were performed, with mesh parameter $3.14 \mathrm{~cm}$, and time step $\Delta t=0.5 \mathrm{~ns}$ :

(a) spatial non-mixed collocation-in-time using third degree Lagrange interpolators,

(b) spatial mixed collocation-in-time using third degree Lagrange interpolators,

(c) spatial non-mixed first order temporal Galerkin,

(d) first order space-time mixed Galerkin.

The relative $H_{\mathrm{div}}^{-1 / 2}$ error of the time domain results with respect to the frequency domain EFIE results is plotted in Fig. 11. In this frequency range, the FD-EFIE does not pick up any internal resonances. Similarly to the case of the cuboid, the accuracy at low frequencies is dominated by the spatial discretization. At high frequencies, it is dominated by the temporal discretization. Over the entire frequency range, the space-time mixed Galerkin scheme yields the most accurate results.

\section{CONCLusions}

In this contribution, a space-time mixed Galerkin discretization for the TD-CFIE has been introduced. It is spatially a mixed Galerkin discretization in that both RWG and BC functions are used in order to obtain an accurate and conforming

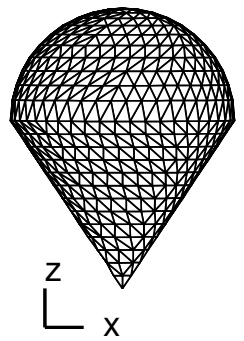

Fig. 10. An ice cream cone discretized using 1230 triangles, with minimal edge length $3.14 \mathrm{~cm}$. The radius of the top hemisphere is $0.3 \mathrm{~m}$, the height of the cone is $0.4 \mathrm{~m}$. The incident electromagnetic field is propagating in the $\mathrm{z}$-direction, while the electric field is polarized along the $\mathrm{x}$-axis.

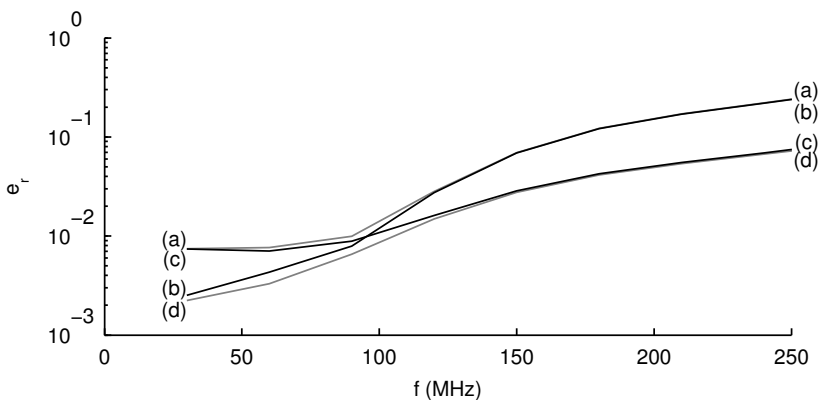

Fig. 11. Relative $H_{\text {div }}^{-1 / 2}$ error of the TD-CFIE simulations for scattering by an ice cream cone with respect to the frequency domain EFIE.

spatial discretization of the TD-CFIE. It is temporally a mixed Galerkin discretization in that different temporal basis and testing functions are used. The temporal mixed discretization has been defined for arbitrary order. The combination of the spatial and temporal mixed Galerkin discretization is essential in order to obtain accurate results over a broad frequency band. This was demonstrated for different numerical examples. Moreover, it has been shown that in order for the space-time mixed Galerkin discretization of the TD-CFIE to be immune to resonances, the TD-EFIE and TD-MFIE contributions need be made consistent by insertion of the appropriate Gram matrices.

Since the interactions in a temporally mixed Galerkin scheme can be identified with interactions in a collocationin-time scheme, the space-time mixed Galerkin discretization can easily be incorporated in existing solvers. Furthermore, the scheme introduced in this contribution is amenable to acceleration by e.g. the PWTD algorithm [10].

\section{REFERENCES}

[1] J. R. Mautz and R. F. Harrington, "H-field, E-field, and combined-field solutions for conducting bodies of revolution," Archiv Elektronik und Uebertragungstechnik, vol. 32, pp. 157-164, Apr. 1978.

[2] S. Rao, D. Wilton, and A. Glisson, "Electromagnetic scattering by surfaces of arbitrary shape," IEEE Trans. Antennas Propag.,, vol. 30, no. 3, pp. $409-418$, may 1982.

[3] B. Shanker, A. Ergin, K. Aygun, and E. Michielssen, "Analysis of transient electromagnetic scattering phenomena using a two-level plane wave time-domain algorithm," Antennas and Propagation, IEEE Transactions on, vol. 48, no. 4, pp. $510-523$, apr 2000.

[4] K. Cools, F. Andriulli, D. De Zutter, and E. Michielssen, "Accurate and conforming mixed discretization of the MFIE," Antennas and Wireless Propagation Letters, IEEE, vol. 10, pp. 528 -531, 2011. 
[5] S. Yan, J.-M. Jin, and Z. Nie, "Improving the accuracy of the secondkind fredholm integral equations by using the buffa-christiansen functions," Antennas and Propagation, IEEE Transactions on, vol. 59, no. 4, pp. $1299-1310$, april 2011

[6] L. Gurel and O. Ergul, "Contamination of the accuracy of the combinedfield integral equation with the discretization error of the magnetic-field integral equation," IEEE Trans. Antennas Propag.,, vol. 57, no. 9, pp. $2650-2657$, sept. 2009.

[7] A. Buffa and S. H. Christiansen, "A dual finite element complex on the barycentric refinement," Comptes Rendus Mathematique, vol. 340, no. 6 , pp. $461-464,2005$.

[8] F. P. Andriulli, K. Cools, H. Bağci, F. Olyslager, A. Buffa, S. Christiansen, and E. Michielssen, "A multiplicative Calderón preconditioner for the electric field integral equation," IEEE Trans. Antennas Propagation, vol. 56, pp. 2398-1930, aug 2008.

[9] K. Cools, F. Andriulli, P. Ylä-Oijala, H. Bagci, D. D. Zutter, and E. Michielssen, "Improving the accuracy of the Calderón preconditioned cfie by using a mixed discretization," in IEEE International Symposium on Antennas and Propagation and CNC/USNC/URSI Radio Science Meeting, Toronto, Canada, July 2010.

[10] W. Chew, E. Michielssen, J. M. Song, and J. M. Jin, Eds., Fast and Efficient Algorithms in Computational Electromagnetics. Norwood, MA, USA: Artech House, Inc., 2001.

[11] A. Yilmaz, J.-M. Jin, and E. Michielssen, "Time domain adaptive integral method for surface integral equations," Antennas and Propagation, IEEE Transactions on, vol. 52, no. 10, pp. 2692 - 2708, oct. 2004.

[12] T. Ha-Duong, "On retarded potential boundary integral equations and their discretisation," in Topics in Computational Wave Propagation, ser. Lecture Notes in Computational Science and Engineering, M. Ainsworth, P. Davies, D. Duncan, B. Rynne, and P. Martin, Eds. Springer Berlin Heidelberg, 2003, vol. 31, pp. 301-336.

[13] I. Terrasse, "Résolution mathématique et numérique des équations de Maxwell instationnaires par une méthode de potentiels retardés," Ph.D. dissertation, Ecole Polytechnique, 1993.

[14] W. C. Chew and J. M. Song, "Gedanken experiments to understand the internal resonance problems of electromagnetic scattering." Electromagnetics, vol. 27, no. 8, pp. 457 - 471, 2007.

[15] B. Shanker, A. Ergin, K. Aygun, and E. Michielssen, "Analysis of transient electromagnetic scattering from closed surfaces using a combined field integral equation," Antennas and Propagation, IEEE Transactions on, vol. 48, no. 7, pp. $1064-1074$, jul 2000.

[16] B. Shanker, M. Lu, J. Yuan, and E. Michielssen, "Time domain integral equation analysis of scattering from composite bodies via exact evaluation of radiation fields," Antennas and Propagation, IEEE Transactions on, vol. 57, no. 5, pp. $1506-1520$, may 2009.

[17] R. E. Hodges and Y. Rahmat-Samii, "The evaluation of MFIE integrals with the use of vector triangle basis functions," Microwave and Optical Technology Letters, vol. 14, no. 1, pp. 9-14, 1997.

[18] Y. Shi, M.-Y. Xia, R.-S. Chen, E. Michielssen, and M. Lu, "Stable electric field TDIE solvers via quasi-exact evaluation of MOT matrix elements," Antennas and Propagation, IEEE Transactions on, vol. 59, no. 2 , pp. $574-585$, feb. 2011.

[19] A. G. Polimeridis and J. R. Mosig, "Complete semi-analytical treatment of weakly singular integrals on planar triangles via the direct evaluation method," International Journal for Numerical Methods in Engineering, vol. 83, no. 12, pp. 1625-1650, 2010.

[20] A. Yucel and A. Ergin, "Exact evaluation of retarded-time potential integrals for the RWG bases," Antennas and Propagation, IEEE Transactions on, vol. 54, no. 5, pp. $1496-1502$, may 2006.

[21] H. Ulku and A. Ergin, "Application of analytical retarded-time potential expressions to the solution of time domain integral equations," Antennas and Propagation, IEEE Transactions on, vol. 59, no. 11, pp. $4123-4131$, nov. 2011

[22] P. J. Davies, "Numerical stability and convergence of approximations of retarded potential integral equations," SIAM Journal on Numerical Analysis, vol. 31, no. 3, pp. pp. 856-875, 1994.

[23] P. Davies and D. Duncan, "Numerical stability of collocation schemes for time domain boundary integral equations," in Computational Electromagnetics, ser. Lecture Notes in Computational Science and Engineering, C. Carstensen, S. Funken, W. Hackbusch, R. H. W. Hoppe, and P. Monk, Eds. Springer Berlin Heidelberg, 2003, vol. 28, pp. 51-67.

[24] S. Sauter and C. Schwab, Boundary Element Methods. Springer, 2010.

[25] O. Ergul and L. Gurel, "Investigation of the inaccuracy of the mfie discretized with the rwg basis functions," in Antennas and Propagation Society International Symposium, 2004. IEEE, vol. 3, june 2004, pp. 3393 - 3396 Vol.3.
[26] D. Weile, G. Pisharody, N.-W. Chen, B. Shanker, and E. Michielssen, "A novel scheme for the solution of the time-domain integral equations of electromagnetics," Antennas and Propagation, IEEE Transactions on, vol. 52, no. 1, pp. $283-295$, jan. 2004.

[27] G. Kaur and A. E. Yilmaz, "Accuracy - efficiency tradeoff of temporal basis functions for time-marching solvers," Microwave and Optical Technology Letters, vol. 53, no. 6, pp. 1343-1348, 2011.

[28] E. van t Wout, H. van der Ven, D. R. van der Heul, and C. Vuik, "A provably stable MoT scheme based on quadratic spline basis functions," in Antennas and Propagation Society International Symposium, 2012. APS '12. IEEE, july 2012.

[29] H. Arda Ülkü, I. Bogaert, K. Cools, F. P. Andriulli, and H. Bağc, "On the low frequency behavior of the mixed discretized time domain magnetic field integral equation," in USNC-URSI National Radio Science Meeting, 2012. USNC-URSI '12. IEEE, july 2012.

[30] A. Buffa and R. Hiptmair, "Regularized combined field integral equations," Numer. Math., vol. 100, no. 1, pp. 1-19, Mar. 2005.

[31] O. Bruno, T. Elling, R. Paffenroth, and C. Turc, "Electromagnetic integral equations requiring small numbers of krylov-subspace iterations," J. Comput. Phys., vol. 228, no. 17, pp. 6169-6183, Sep. 2009.

[32] A. Buffa and P. Ciarlet, "On traces for functional spaces related to Maxwell's equations part I: An integration by parts formula in Lipschitz polyhedra," Mathematical Methods in the Applied Sciences, vol. 24, no. 1, pp. 9-30, 2001.

[33] J. Van Bladel, Electromagnetic Fields, 2nd ed., ser. IEEE press series on electromagnetic wave theory. Wiley-IEEE Press, 2007.

[34] R. F. Harrington, Time-harmonic Electromagnetic Fields. McGraw-Hill, 1961.

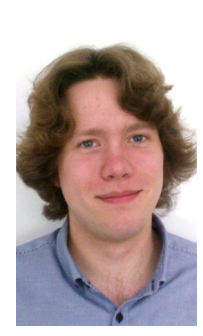

Yves Beghein received the M.Sc.Eng. degree in physical engineering from Ghent University, Belgium, in 2011. His master's dissertation dealt with the efficient modeling of scattering by chiral media. $\mathrm{He}$ is currently pursuing a Ph.D. degree at the Electromagnetics Group at Ghent University, under the advisorship of Prof. Kristof Cools and Prof. Daniël De Zutter. His research focusses on both time domain and frequency domain boundary element methods, their stable and accurate discretizations, and efficient solution methods.

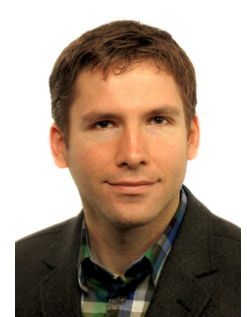

Kristof Cools received the M. Eng. degree in Physical Engineering from Ghent University, Belgium, in 2004. In August 2004, he joined the Electromagnetics Group at Ghent University. He received the Ph.D. degree from Ghent University in 2008 , under the advisership of Prof. F. Olyslager and Prof. E. Michielssen. In 2009, he was awarded the Young Scientist Best Paper award at the International Conference on Electromagnetics and Advanced Applications. In 2011, he was a visiting research fellow at TELECOM Bretagne. In November 2011, he took up a position as Lecturer in Computational Electromagnetics at the University of Nottingham. His research focuses on the spectral properties of the boundary integral operators of electromagnetics, on stable and accurate discretization schemes for frequency and time domain boundary element methods, on domain decomposition techniques, and on the implementation of algorithms from computational physics for high performance computing. 


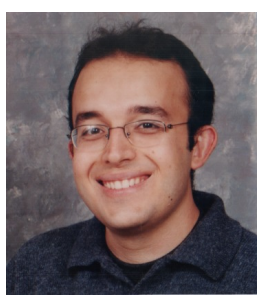

Hakan Bağcı received the B.S. degree in Electrical and Electronics Engineering from the Bilkent University, Ankara, Turkey, in June 2001 and the M.S. and Ph.D. degrees in Electrical and Computer Engineering from the University of Illinois at Urbana-Champaign (UIUC), Urbana, in August 2003 and January 2007, respectively. From June 1999 to July 2001, he worked as an Undergraduate Researcher at the Computational Electromagnetics Group, Bilkent University. From August 2001 to December 2006, he was a Research Assistant at the Center for Computational Electromagnetics and Electromagnetics Laboratory, UIUC. From January 2007 to August 2009, he worked as a Research Fellow at the Radiation Laboratory, University of Michigan. In August 2009, he joined the Division of Physical Sciences and Engineering at the King Abdullah University of Science and Technology (KAUST) as Assistant Professor of Electrical Engineering. His research interests include various aspects of computational electromagnetics with emphasis on time-domain integral equations and their fast marching-on-in-time-based solutions, well-conditioned integralequation formulations, and development of fast hybrid methods for analyzing statistical EMC/EMI phenomena on complex and fully loaded platforms. Dr. Bağc1 was the recipient of the 2008 International Union of Radio Scientists (URSI) Young Scientist Award and the 20042005 Interdisciplinary Graduate Fellowship from the Computational Science and Engineering Department, UIUC. His paper titled "Fast and rigorous analysis of EMC/EMI phenomena on electrically large and complex structures loaded with coaxial cables" was one of the three finalists (with honorable mention) for the 2008 Richard B. Schulz Best Transactions Paper Award given by the IEEE Electromagnetic Compatibility Society. He authored and co-authored three finalist papers and another paper, which is awarded honorable mention, in the student paper competitions at the 2005, 2008, and 2010, IEEE Antennas and Propagation Society International Symposiums.

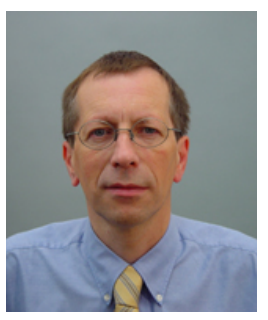

Daniël De Zutter was born in 1953 . He received his M. Sc. Degree in electrical engineering from the University of Gent in 1976. In 1981 he obtained a Ph. D. degree and in 1984 he completed a thesis leading to a degree equivalent to the French Aggrégation or the German Habilitation. He is now a full professor of electromagnetics. His research focusses on all aspects of circuit and electromagnetic modelling of high-speed and high-frequency interconnections and packaging, on Electromagnetic Compatibility (EMC) and numerical solutions of Maxwells equations. As author or co-author he has contributed to more than 180 international journal papers (cited in the Web of Science) and 200 papers in conference proceedings. In 2000 he was elected to the grade of Fellow of the IEEE. He was an Associate Editor for the IEEE Microwave Theory and Techniques Transactions. Between 2004 and 2008 he served as the Dean of the Faculty of Engineering of Ghent University and is now the head of the Department of Information Technology. 